\title{
Optoelectronic evaluation of the nanostructuring approach to chalcopyrite-based intermediate band materials
}

\author{
D. Fuertes Marrón a,*, E. Cánovas ${ }^{\mathrm{a}}$, M.Y. Levy ${ }^{\mathrm{a}}$, A. Martí ${ }^{\mathrm{a}}$, A. Luque ${ }^{\mathrm{a}}$, M. Afshar ${ }^{\mathrm{b}}$, J. Albert ${ }^{\mathrm{b}}$, S. Lehmann ${ }^{\mathrm{b}}$, \\ D. Abou-Ras ${ }^{\text {b }}$, S. Sadewasser ${ }^{\text {b }}$, N. Barreau ${ }^{\mathrm{c}}$ \\ a instituto de Energra Solar-ETSIT, UPM. Ciudad Universitaria s.n., 28040 Madił, Spain \\ b Heinhoitz-Zentırm Berin für Materialen und Energie, Glienicker Str. 100, 14109 Berlin, Geımany \\ 'Institut des Matériaux Jean Rauxel (IMN)-UMR 6502, Université de Nantes, CNRS, 2 ize de la Houssinière, BP 32229, 44322 Nantes cedex 3, France
}

\section{A R T I C L E I N F O}

Keywords:

Chalcopyrite

Nanostructures

Solar cells

\begin{abstract}
A B S T R A C T
Nanostructured chalcopyrite compounds have recently been proposed as absorber materials for advanced photovoltaic devices. We have used photoreflectance (PR) to evaluate the impact of interdiffusion phenomena and the presence of native defects on the optoelectronic properties of such materials. Two model material systems have been analyzed: (i) thin layers of $\mathrm{CuGaSe}_{2}\left(E_{\xi}=1.7 \mathrm{eV}\right)$ and $\mathrm{CuInSe}_{2}(1.0 \mathrm{eV})$ in a wide/low/wide bandgap stack that have been grown onto GaAs $(001)$ substrates by metalorganic chemical vapor deposition (MOCVD); and (ii) thin $\ln _{2} S_{3}$ samples $\left(E_{g}=2.0 \mathrm{eV}\right.$ ) containing small amounts of $\mathrm{Cu}$ that have been grown by co-evaporation (PVD) intending to form $\mathrm{Cu}_{\mathrm{x}}\left[\mathrm{n}_{y} \mathrm{~S}_{z}\left(\mathrm{E}_{\mathrm{g}} \sim 1.5 \mathrm{eV}\right)\right.$ nanoclusters into the $\ln _{2} \mathrm{~S}_{3}$ matrix. The results have been analyzed according to the third-derivative functional form (TDFF). The valence band structure of selenide reference samples could be resolved and uneven interdiffusion of $\mathrm{Ga}$ and $\mathrm{In}$ in the layer stack could be inferred from the shift of PR-signatures. Hints of electronic confinement associated to the transitions at the low-gap region have been found in the selenide layer stack. Regarding the sulphide system, $\left[n_{2} S_{3}\right.$ is characterized by the presence of native deep states, as revealed by PR. The defect structure of the compound undergoes changes when incorporating $\mathrm{Cu}$ and no conclusive result about the presence of ternary clusters of a distinct phase could be drawn. Interdiffusion phenomena and the presence of native defects in chalcopyrites and related compounds will determine their potential use in advanced photovoltaic devices based on nanostructures.
\end{abstract}

\section{Introduction}

The use of nanostructured thin-film chalcopyrite absorbers for high-efficiency photovoltaic applications has been recently proposed [1]. The driving force behind this interest is the possibility of combining the potential and unique properties of these compounds for the realization of thin-film solar cells with the domains of quantum-size effects and novel designs of related devices. Among such designs, the realization of a quantum-dot, intermediate-band solar cell based on thin-film technology and related materials is currently under study. The architecture of the device will consist of nanostructures made of an absorbing material embedded into a semiconducting matrix of a wider bandgap. The discrete electronic levels of ideally zero-dimensional quantum dots form minibands within the bandgap of the host compound. These minibands provide an intermediate band isolated from the valence and conduction band of the host by an

\footnotetext{
* Corresponding author.

E-mail address: dfuerteseies-def.upm.es (D. Fuertes Marrón).
}

energy range of zero density of states, as originally proposed by Luque and Marti [2]. The device is completed with two emitters that selectively extract electrons and holes at either side of the absorber. In this way, three absorption onsets are expected at photon energies corresponding to transitions from the valence band to the intermediate band, from the intermediate to the conduction band, and from the valence to the conduction band. leading to an increase of the generated photocurrent. The proper use of the emitters and the isolation of the intermediate band material from the contacts ensure that the expected voltage delivered by the device will be limited by the main gap of the material and not by any of the sub-bandgaps associated to the intermediate band. The overall balance is an increased efficiency of energy conversion [3|.

Chalcopyrites lead the efficiency ranking of thin-film technologies and therefore appear as potential candidates for advanced designs [4]. Different approaches to the growth of nanostructured Cu-containing chalcogenides have been reported [5], many of them based on solvothermal reactions leading to the formation of powders. These methods, despite the fact of having demonstrated intrinsic properties associated to reduced dimensions, appear 
nevertheless inadequate to implement nanostructured chalcopyrites into solid-state devices. This inadequacy results from the absence of a suitable semiconductor matrix that may be used to embed the nanostiuctured material. In particular, a suitable semiconductor matrix must have a larger bandgap than the nanostructured material, providing the confining potential energy barrier in the form of a suitable band offset. Procedures for the growth of chalcopyrite nanocrystals and mesoscopic clusters in the form of thin films have also been proposed that allow for the embedding step [6]. The embedding process of chalcopyrite nanocrystals into a barrier material, typically a related binary chalcogenide or a widegap ternary counterpart, can in principle be accomplished either during the growth of the nanostructured material itself or following a sequential process, like in the case of conventional quantum well structures. In any case, the quality of the interface between the buried material and the barrier matrix appears critical, as it will determine (i) the electronic properties of the metallurgical junction between both materials, and (ii) the profile of the confining potential. Elemental interdiffusion processes between components of barrier and buried compounds will prevent the formation of abrupt interfaces and will determine the profile of the potential barrier. Interdiffusion does indeed govern the alloying in the chalcopyrite system $\mathrm{Cu}(\mathrm{Ga}, \mathrm{In})(\mathrm{S}, \mathrm{Se})_{2}$ as used for high efficiency solar cells [ $4 \mid$ and is therefore expected to play a central role in the fabrication of nanostructured interfaces between chalcopyrite compounds of two different types. Additionally, chalcopyrites and related binary compounds show high concentrations of native defects of different types, despite their overall relative benign character for efficient devices. In this contribution, results of the optoelectronic characterization of nanostructured chalcopyrites will be presented, with the aim of determining the impact of these two important issues: (i) interdiffusion phenomena between elements of the matrix and the embedded nanostructure; and (ii) the presence of native defects in the nanostructured material.

\section{Experimental}

We have devised two different nanostructured material systems to study separately interdiffusion phenomena, occurring at standard processing temperatures, and the impact that the presence of native defects may have on the optoelectronic properties of the samples. For the study of interdiffusion phenomena, epitaxial selenide samples in the form of a quantum well, with alternating layers of different compositions and wide/ low/wide bandgaps, have been grown by metalorganic chemical vapor deposition (MOCVD) onto GaAs substrates [7]. For the study of native defects and their effects on the optoelectronic properties, we have analyzed evaporated microcrystalline ${ }_{n} n_{2} S_{3}$ samples grown at low temperature that included reduced amounts of $\mathrm{Cu}$ during processing, in an attempt to form nanocrystals of $\mathrm{Cu}_{x}\left[n_{y} S_{z}\right.$ within the $\left[n_{2} S_{3}\right.$ matrix.

\subsection{Selenide samples}

MOCVD-grown samples have been processed at a substrate temperature of $500^{\circ} \mathrm{C}$ onto clean semi-insulating, epi-ready GaAs(001) wafers $\left(500 \mu \mathrm{m}, \mathrm{EPD}<3 \times 10^{3} \mathrm{~cm}^{-2}\right.$, Wafertechnology Ltd.). Details on the growth system and procedures can be found in Ref. [6] and references therein. A triple-layer structure has been analyzed consisting of $\mathrm{GaAs}($ substrate)/CuGaSe$/$ CulnSe $/ \mathrm{CuGaSe}_{2}$. In addition, two single-layer samples, namely $\mathrm{GaAs}_{\mathrm{Cu}} / \mathrm{Cu} \mathrm{Se}_{2}(1.0 \mathrm{eV})$ and $\mathrm{GaAs}_{\mathrm{CuGaSe}}\left(E_{\mathrm{g}}=1.7 \mathrm{eV}\right)$ served as reference. For both the reference samples and the layer stack, each single chalcogenide layer had a nominal thickness of $\sim 100 \mathrm{~nm}$, confirmed by scanning (SEM, LEO-Gemini 1530) and transmission electron microscopy (TEM, Zeiss Libra operated at $200 \mathrm{kV}$ equipped with a Thermo Noran EDX analysis unit) cross-sectional analysis. The samples showed $c(001)$ orientation, according to $\mathrm{X}$-ray diffraction analysis and electron channeling experiments [8]. Fig. 1 shows a cross-sectional SEM image of the CuInSe 2 reference sample ( $70^{\circ}$ off-normal), revealing the closed interface between the substrate and the layer and the grooves resulting from the typical faceting into polar $\left\{\begin{array}{lll}1 & 1 & 2\end{array}\right\}$-planes of the free chalcopyrite surface [9].

\subsection{Sulphide samples}

Polycrystalline PVD-grown samples of $\left[n_{2} S_{3}\left(E_{g}=2.0 \mathrm{eV}\right)\right.$ have been grown onto Mo-coated. Na-free glass (Corning 7059) at a low substrate temperature $\left(200^{\circ} \mathrm{C}\right.$ ) by co-evaporation. Bare $\ln _{2} S_{3}$ samples served as a reference, to be compared to identical samples that included a small amount of Cu during the process (tota] $[\mathrm{Cu}] /([\mathrm{Cu}]+[\mathrm{ln}])$ ratio $\sim 2-3$ at\% according to EDX analysis). The process sequence followed for the metals of the $\mathrm{Cu}$-containing sample was

\section{- In (55 nm)-Cu (1 nm)-In (60 nm)-Cu (1 nm)-In (65 nm)-Cu (1 nm)-[n (70 nm)}

with constant supply of $S$ in excess over the nominal stoichiometry during the entire process, for a total thickness of $\sim 250 \mathrm{~nm}$. Identical samples grown on uncoated glass showed different coloring of the Cu-containing (orange) and Cu-free semitransparent samples (yellow), not shown.

\subsection{Photoreflectance setup and basics}

Photoreflectance (PR) belongs to the family of modulation spectroscopy techniques able to probe the changes in the optoelectronic properties of semiconducting samples upon the application of a perturbing electric field. For this purpose, a monochromatized light beam (in our case from a $150 \mathrm{~W}$ halogen lamp) is focused on the surface of the sample in near-normal incidence, and the reflected beam is collected with optical lenses

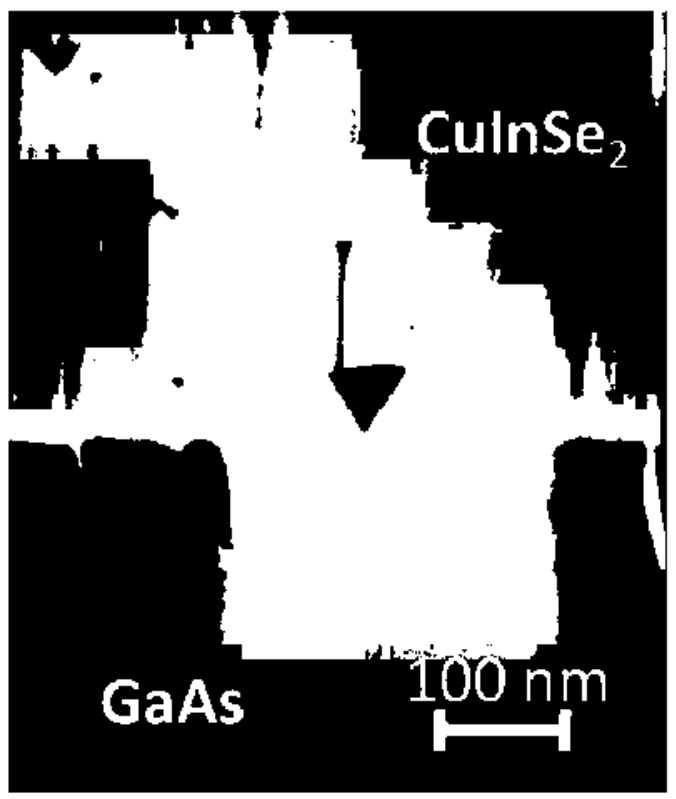

Fig. 1. Cross-5ectional SEM $70^{\circ}$ tilted view of an epitaxial Culn5e, layer grown by MOCVD at $500^{\circ} \mathrm{C}$ on GaAs $\left(\begin{array}{lll}0 & 0 & 1\end{array}\right)$ 
and focused on a photodetector (Si- and Ge-photodiodes for different wavelength ranges). Superimposed onto the monochromatic light spot at the sample, a laser beam (17 $\mathrm{mW}$. HeNe at $632.8 \mathrm{~nm}$ ) chopped at $777 \mathrm{~Hz}$ acts as a pump-beam. The pump beam hits the sample surface with an incidence angle of $\sim 75^{\circ}$ with respect to the normal. The high absorption coefficient of chalcopyrite compounds plus the open angle of incidence of the pump beam limit the region of the sample being probed in PR to the near surface. The reflectance signal modulated at the reference frequency and collected at the detector is amplified by a lock-in amplifier (Stanford Instruments). Basics of the technique can be found in Ref. [10] and references therein. Modulation spectroscopy has been used for the characterization of chalcopyrite compounds since the works of Shay and Tell [11] with particular emphasis on the determination of the valence band structure of such compounds. More recently, Shirakata and Chichibu [12] have reported excellent photoreflectance (PR) studies of epitaxial layers belonging to the $\mathrm{Cu}(\mathrm{Al}, \mathrm{Ga}, \mathrm{In})(\mathrm{S}, \mathrm{Se})_{2}$ system. What follows is just a brief summary of the basics of the technique.

The analysis of the spectra obtained from PR measurements can give detailed information about the electronic structure of the sample investigated. The main effect of the modulated pumpbeam is to modify in a periodic manner the internal electric fields of the sample. The latter are associated with built-in electrostatic potentials at interfaces, free surfaces and, eventually, at localised and electrically active defects. The variation in the internal electric fields has in turn a direct impact on the values of the dielectric function, ultimately responsible of the reflectivity of the sample that will undergo a modulated variation following the perturbing light beam. The changes of the dielectric constants of the samples are most prominent around critical points of the electronic structure or van Hove singularities, which are associated to sharp variations in the density of states. The lineshape of the PR spectra reflects thus the variations of the dielectric function of the sample at such points and will depend on the character of the critical point involved. In contrast to other possible modulated perturbations, like thermal oscillations or piezo-strain, the perturbing electric filed breaks the translational symmetry associated to the Bloch functions of carriers in the upper electronic bands. Aspnes [13] analyzed the effect of translational-symmetry-preserving- vs. translational symmetrybreaking-perturbations, setting the basics for the analysis of the lineshapes of modulated spectra. The so-called third derivative functional form (TDFF) is the basic tool for PR analysis:

$\frac{\Delta R}{R}=R e\left\{\sum_{j}\left[C_{j} e^{i \theta_{j}}\left(E-E_{g, j}+i \Gamma_{j}\right)^{-m_{j}}\right]\right\}$

where it is stated that the PR signal is given by the real part of the sum over the different $j$ critical points $E_{g j}$ involved of a functional that includes an amplitude factor $C$, a phase $\theta$, a broadening parameter $\Gamma$, and an exponential term $m$ that accounts for the type of critical point and the related order of the derivative, which can have only certain values with physical meaning:

- $m=2.5$, corresponds to the third derivative of a 3-D (parabolic) critical point;

- $m=3$, is associated to a 2-D critical point;

- $m=3.5$, is associated to a 1-D critical point; and

- $m=2$, corresponds to the first derivative of a Lorentzian; associated to excitonic transitions and/or bound states where the carriers cannot be displaced following the perturbing field.

The cases $m=2.5,3$, and 3.5 (third derivative functional) correspond to situations in which there is no electronic confine- ment of the carriers associated to the transitions, in contrast to the case $m=2$. The TDFF will be used in the analysis of our samples aiming, on the one hand, at the identification of critical points associated to the chalcopyrite phases that will thus reveal their presence and, on the other hand, at the determination of the eventual confined character of the carriers involved in the corresponding transitions. The reader is referred to Ref. [13] for further details on the nature and effects of the different parameters included in the TDFF on the fitting results.

\section{Results}

\subsection{The selenide system}

Fig. 2 shows a cross-sectional EDX-compositional image obtained from the GaAs/CuGaSe $/ \mathrm{CuInSe}_{2} / \mathrm{CuGaSe}_{2}$ stack. The upper left figure is the TEM picture of the section and the rest of the panels correspond to L-emission line intensity mapping of the constituent elements $\mathrm{Cu}, \mathrm{Ga}, \mathrm{Se}, \mathrm{As}$, and In as measured by EDX. The image shows a well resolved GaAs/chalcopyrite interface. Two faceting-induced grooves at the front surface of the layer are readily visible. Both $\mathrm{Cu}$ - and Se-distributions appear homogeneous throughout the entire sample thickness. However, $G a$ and In appear unevenly distributed over the entire sample thickness. The presence of both $\mathrm{Ga}$ and $\mathrm{In}$ in the layer indicates that interdiffusion and alloying of both group-[II elements in the cation-sublattice of the chalcopyrite structure has taken place at processing temperatures, as expected. The uneven distribution of either element is in agreement with the compositional growth sequence: at the central part of the layer a higher In-concentration, corresponding to Ga-depletion, is observed. The upshot is a sample with a compositional gradient, at least in the direction normal to the surface (lateral inhomogeneities of the In-content can indeed be also inferred from the corresponding [n-map). Although no sharp nor well defined interface between $\mathrm{Ga}$ - and In-containing phases is observed, the varying $[\mathrm{Ga}] /([\mathrm{Ga}]+[\mathrm{In} \mid)$ ratio will result in a corresponding variation of the bandgap with respect to the growth direction.

Fig. 3 shows room temperature PR spectra (open dots) of the

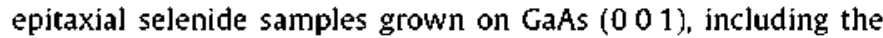
two reference samples of $\mathrm{CuGaSe}_{2}$ (upper panel) and $\mathrm{CuInSe}_{2}$ (lower panel). The spectra have been fitted according to the TDFF (Eq. (1)) and the corresponding fits are shown as solid lines. Analysis of the spectra reveal that there are three critical points, denoted $A, B$, and $C$ in the figure, which account for the three bandgaps associated to the three-fold valence band characteristic of chalcopyrite compounds. The upper part of the valence band of Cu-containing chalcopyrites consists mainly of hybridized states from $\mathrm{Cu}-3 \mathrm{~d}$ and chalcogen p-states (either $\mathrm{S}$ or Se). The three-fold valence band stems from the splitting due to spin-orbit interaction and to the crystal field associated to the tetragonal distortion in the chalcopyrite structure (a non-cubic lattice with $c \neq 2 a)[11]$. For CulnSe $e_{2}$, the tetragonal distortion is small and larger than unity $(c>2 \mathfrak{a})$, whereas for $\mathrm{CuCaSe}_{2}$, the distortion is larger in magnitude than for CuInSe $e_{2}$ and smaller than unity ( $c<2 a$ ). The composition for which $c=2 a$ corresponds to a [Ga]/ ([Ga $\mid+[\mathrm{ln} \mid)$ ratio of $\sim 0.25$, according to Vegard's rule and supported by experimental studies [14].

The energy of the critical points obtained from the TDFF fittings of the spectra of the reference $\mathrm{CuGaSe}_{2}$ and CuInSe samples are in good agreement with reported values of the three gaps and the corresponding splittings [11]. These values are summarized in Table 1 . For CulnSe $\mathrm{S}_{2}$ the fundamental gap A appears at $1.01 \mathrm{eV}$, partially overlapped by the second gap $B$, resulting from the crystal-field splitting, at $1.03 \mathrm{eV}$. The 

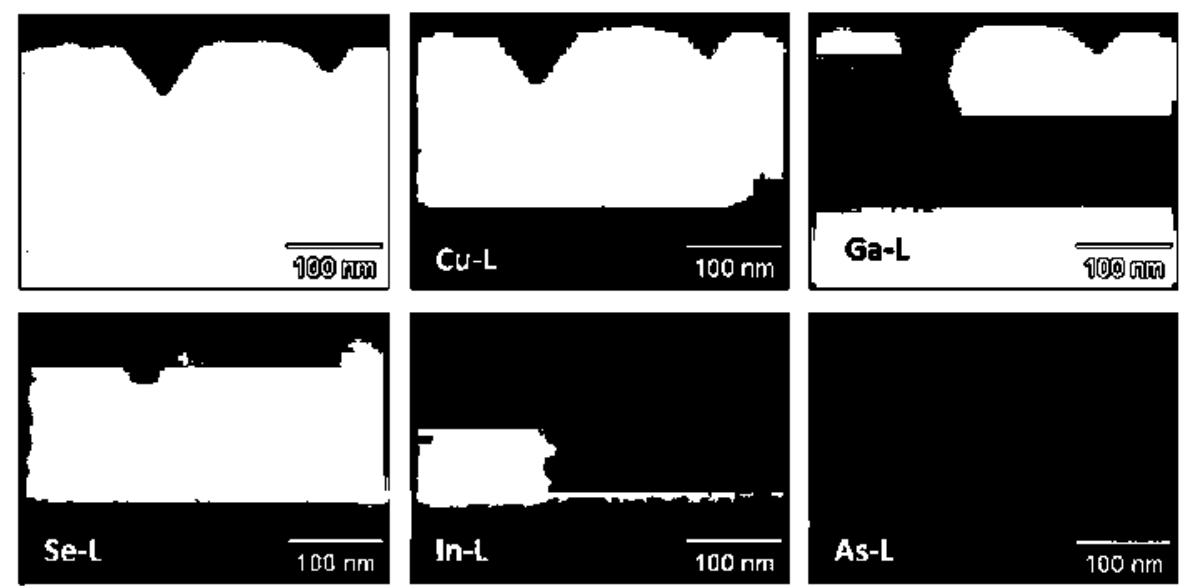

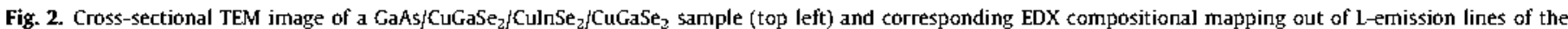
constituent elements.

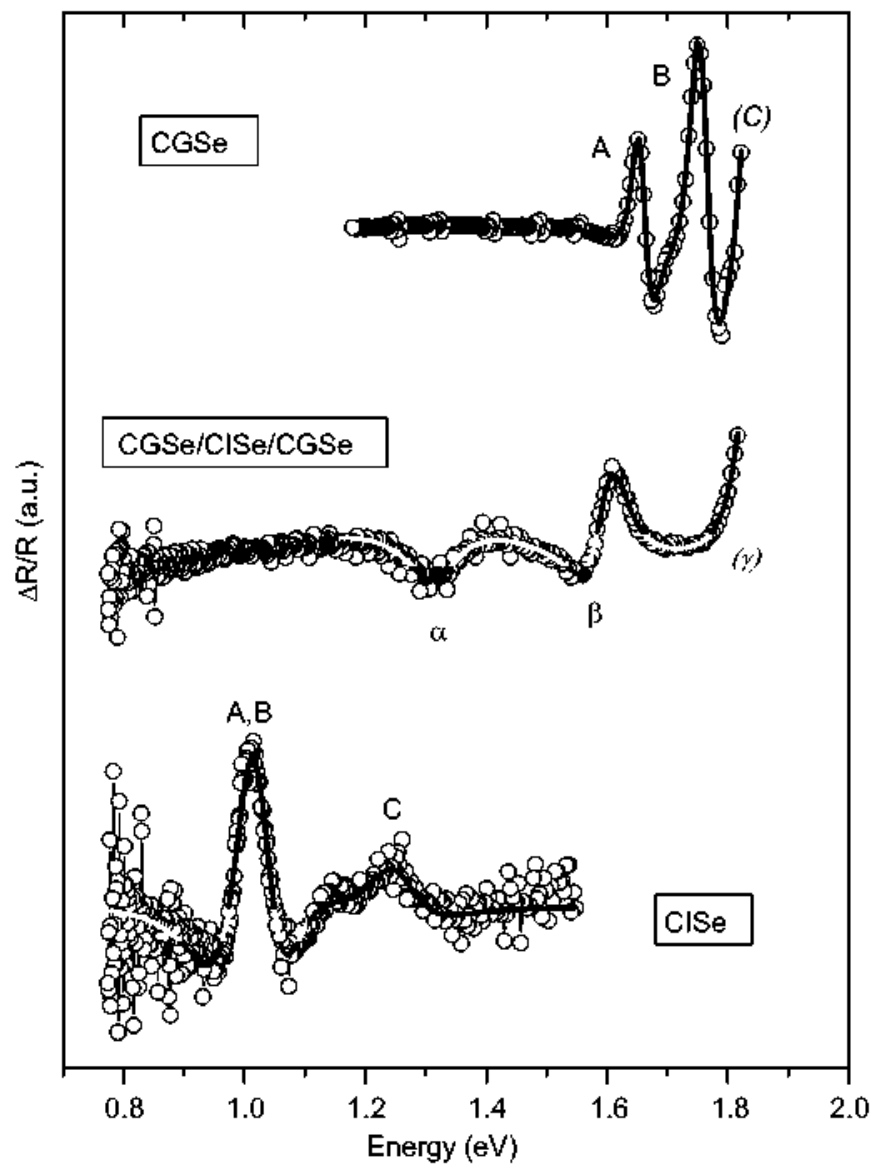

Fig. 3. PR spectra (open dots) of epitaxial selenide layers on GaAs ( 000 1): $\mathrm{CuGaSe}_{2}$ (top). CulnSe $\mathrm{e}_{2}$ (bottom), and CuGaSe $f_{2}$ CulnSe $/ \mathrm{CuGaSe}_{2}$ stack. Solid lines represent TDFF fittings with parameters as summarized in Table 1.

corresponding broadening parameters are relatively large, around $80 \mathrm{meV}$, but still a TDFF-fit with a single critical point did not yield a satisfactory result. The third gap $C$ (spin-orbit) is well resolved at $1.25 \mathrm{eV}$. For $\mathrm{CuGaSe}_{2}$, transitions $\mathrm{A}$ and $\mathrm{B}$ at 1.66 and $1.76 \mathrm{eV}$ appear well resolved, although transition $\mathrm{C}$ is only partly visible in the range accessible due to the use of an optical filter before the detector. The energies of $A$ and $B$ transitions are in accord with reported data [11]. For both reference samples the m-factors obtained from best fits to TDFF are consistent with transitions involving free carriers around 3-D critical points. This is characteristic of bulk material, excluding the presence of bound states, and in agreement with photoluminescence results, where excitonic emission from chalcopyrite thin films could only be resolved at low temperatures with no excitonic signatures at room temperature [15]. In none of the samples could the fundamental gap of GaAs be resolved from the PR spectra. This may be explained by considering that no perturbing field effectively modulates the buried interface of the chalcopyrite layer with the substrate.

In the case of the layer stack, three apparent PR features are readily visible in Fig. 3, which are denoted by $\alpha, \beta$, and $\gamma$. No CISerelated signatures at about $1.00 \mathrm{eV}$ could be found and the highenergy range in the plot appears different from that of the $\mathrm{CuGaSe}_{2}$ reference. Two different fits of the spectrum of the layer stack sample have been performed. In a first attempt, we have limited the number of critical points to three, accounting for the conspicuous features of the spectrum. The corresponding parameters are summarized in Table 1 , together with results from the fittings of the reference samples. According to this procedure, transition $\alpha$ appears at $1.32 \mathrm{eV}$, well above any transition observed for the case of CulnSe $e_{2}$, whereas transition $\beta$ appears at $1.60 \mathrm{eV}$, below any of the transitions related to pure CuGaSe . We cannot conclude with certainty about the position of transition $\gamma$. To explain the origin of transitions $\alpha$ and $\beta$ in the layer stack, the authors recall that non-negligible amounts of $\mathrm{Ga}$ and In had been detected by means of EDX throughout the entire layer thickness. The compositional variation leads to corresponding shifts in the energy positions of the bandgaps, and thus to the energies of the related critical points accessible by PR. These energies can be calculated from empirical expressions relating the energy gaps of the alloys and their actual composition, which include bowing coefficients [16]:

$$
\begin{aligned}
& E_{g_{A}}=1.010+0.626 x-0.167 x(1-x) \\
& E_{g .8}=1.006+0.702 x-0.166 x(1-x) \\
& E_{g . C}=1.236+0.674 x-0.151 x(1-x)
\end{aligned}
$$

where $E_{g, A}, E_{g, B}$, and $E_{g, C}$ (in eV) correspond to transitions $A, B$, and $C$, respectively, and $x$ represents the Ga-fraction of the overall group-]ll content in the selenide system ( $x=0$ for CulnSe ${ }_{2} ; x=1$ for $\mathrm{CuGaSe}_{2}$ ). Substituting $1.32 \mathrm{eV}$ for $E_{g, A}$, i.e., assuming that the lowest-energy signature of the PR spectrum corresponds to the fundamental gap for a given Ga-content $x$ in the equations, a Gacontent of 55.3 at\% is obtained. The corresponding crystal-fieldand spin-orbit-related transitions should then read $E_{g B}=1.35 \mathrm{eV}$ 
Table 1

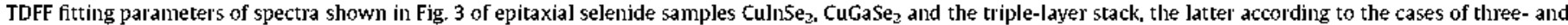
six critical points (CPS).

\begin{tabular}{|c|c|c|c|c|c|}
\hline & $\mathrm{CulnSe}_{2}$ & $\mathrm{CuGaSe}_{2}$ & $\mathrm{CuGaSe}_{2} / \mathrm{CulnSe} / \mathrm{CuGaSe}$ (3-CPs) & \multicolumn{2}{|c|}{$\mathrm{CuGaSe}_{2} / \mathrm{CulnSe} / \mathrm{CuGaSe}$ (6-CPs) } \\
\hline$E_{R A}(\mathrm{eV})$ & 1.015 & 1.659 & $(\alpha) 1.317$ & 1.310 & 1.604 \\
\hline$E_{g B}(\mathrm{eV})$ & 1.035 & 1.760 & (B) 1.597 & 1.345 & 1.670 \\
\hline$E_{g C}(e V)$ & 1.250 & $(1.865)$ & $(\gamma)(1.902)$ & 1.573 & $(1.925)$ \\
\hline$\Gamma_{A}(\mathrm{meV})$ & 85 & 28 & (a) 83 & 72 & 63 \\
\hline$\Gamma_{e}(\mathrm{meV})$ & 79 & 35 & 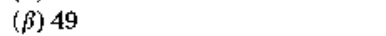 & 70 & 178 \\
\hline$\Gamma_{C}(\mathrm{mev})$ & 85 & (33) & $(\gamma)(45)$ & 52 & $(56)$ \\
\hline$m_{A}$ & 2.49 & 2.74 & (a) 2.03 & 1.84 & 2.67 \\
\hline$m_{e}$ & 2.50 & 2.54 & (B) 2.12 & 2.02 & 2.85 \\
\hline$m_{C}$ & 2.49 & $(2.45)$ & ( $\gamma)(2.25)$ & 1.98 & $(2.65)$ \\
\hline
\end{tabular}

and $E_{g, c}=1.57 \mathrm{eV}$, respectively, according to Eq. (2). However, the energies of the critical points obtained from the TDFF fit (Table 1) do not agree with these figures. A second fit has been attempted, allowing for six critical points in the spectrum. Six transitions would account for the three-fold valence band structure of two different compounds, differing in their composition. We have assumed that $\alpha$-signature is related to transitions associated with a low-gap alloy, whereas $\beta$-signature includes contributions of a widegap alloy. Calculating for $E_{g, A}=1.60 \mathrm{eV}$, the corresponding Ga-content of the Ga-rich alloy is 95.0 at\%. For such Gaconcentration, transitions $B$ and $C$ should appear at $E_{g, B}=1.66 \mathrm{eV}$ and $E_{g . c}=1.87 \mathrm{eV}$, according to Eq. (2). The results of the sixcritical-point fit, as performed with the corresponding energies as free parameters (i.e. with no imposed constraints to the inferred values) are summarized in Table 1 and the solid line in Fig. 3 for the layer stack shows the fitting curve. They are in good agreement with the calculated energy transitions with a significant deviation only in the value of $\gamma$-signature, likely attributable to the fact that it has just been partly resolved in the measurement. We thus conclude that the use of six critical points in the analysis of the PR spectrum of the layer stack is justified, since it provides physically meaningful results in accord with the experimental data.

ln light of these results, the interdiffusion process seems to be uneven with respect to the diffusivities of $G a$ and $\mathrm{ln}$ species. Interpreting transitions $\alpha$ and $\beta$ as stemming from In-containing and Ga-containing compounds, respectively, it is concluded that incorporation of Ga into the In-containing compound is much more effective than the corresponding incorporation of ln into the Ga-containing compound. Afshar estimated the diffusion length $[2 \sqrt{ }(D t)]$ of $\mathrm{Ga}$ and In out of the compositional profiles as measured by EDX in similar samples with an inverted structure,

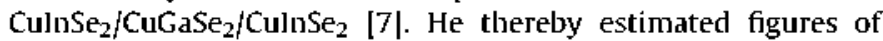
$19 \pm 3 \mathrm{~nm}$ for out diffusion of $\mathrm{Ga}$ into $\mathrm{CulnSe}_{2}$ and $22 \pm 2 \mathrm{~nm}$ for the corresponding In-outdiffusion into $\mathrm{CuGaSe}_{2}$. This result is consistent with the observed features, although the origin and amount of diffusing species in the inverted structure is not directly transferable to our case (double Ga-stiucture, apart from the substrate, and thus double amount of $\mathrm{Ga}$ at the source of Gadiffusion). In any case, assuming that the observed PR transitions correspond to the extrema of the bandgaps present in the multilayer sample, we can represent the situation of the sample with respect to the nominal case in a common band-diagram. Fig. 4 represents the expected band-diagram for the triple-layer sample and the actual band alignment, according to the compositional results and the observed PR transitions.

lnterestingly, best fits following the TDFF are obtained, both for the case of three- and six critical points, with exponent $m$-factors around 2.0 for transitions associated with the low-gap alloy. According to the physical interpretation of the $m$-values described in Section 2.3, these transitions are associated to bound

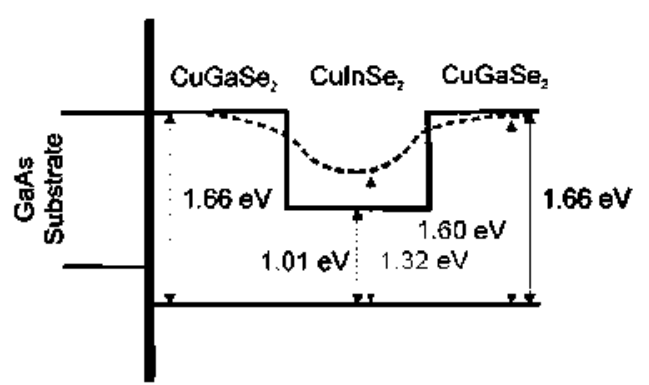

Fig. 4. 5chematic band-diagram of the multi-layer stack. Black solid lines denote the nominal structure, the dashed line represents the actual conduction band profile according to the interpretation of PR results.

states (i.e., $m=2.0$ denotes electronic confinement in the structures). However, we excluded before the possibility of excitonic emission at room temperature in bulk samples. Therefore, these results seem to indicate that electronic confinement can be achieved out of chalcopyrite nanostructures. Nevertheless, it appears clear that cation interdiffusion will ultimately define the potential profiles (most likely in the three dimensions and not only in the direction normal to the plane of growth, according to compositional results) that photogenerated carriers will see.

\subsection{The sulphide system}

Fig. 5 shows the PR spectra and the corresponding TDFF fittings of the $\left[n_{2} S_{3}\right.$ reference and the $\mathrm{Cu}$-containing samples. The fitting has been done with varying number of critical points and the figure shows the best fits obtained for both types of samples. The parameter values of the corresponding fits are summarized in Table 2.

An apparent single feature around $1.4 \mathrm{eV}$ in the reference $\left[n_{2} \mathrm{~S}_{3}\right.$ sample (upper panel) is composed of two contributions at 1.38 and $1.52 \mathrm{eV}$, labeled $\mathrm{a}$ and $\mathrm{b}$ in the figure. There appears $\mathrm{a}$ significant difference between these two signatures with respect to the broadening parameter $\left(\Gamma_{a} \sim 41 \mathrm{meV}, \Gamma_{a} \sim 210 \mathrm{meV}\right)$. This difference is not fully understood and may indeed point to the presence of additional critical points at still higher energies. The Cu-containing sample is best fit by three transitions (primed labels) at $0.90,1.48$ and $1.53 \mathrm{eV}$, the general appearance of the spectrum being noticeably different from that of the reference sample. The $m$-values of all critical points are approximately equal to 2, indicating that there exists electronic confinement associated to the transitions involved. In the case of the reference sample, neither $a$ nor $b$ can be associated to the fundamental gap of $\ln _{2} S_{3}$ (at $\sim 2.0 \mathrm{eV}$, likely indirect and therefore, in principle, PR-silent). They are thus attributed to the presence of deep states 


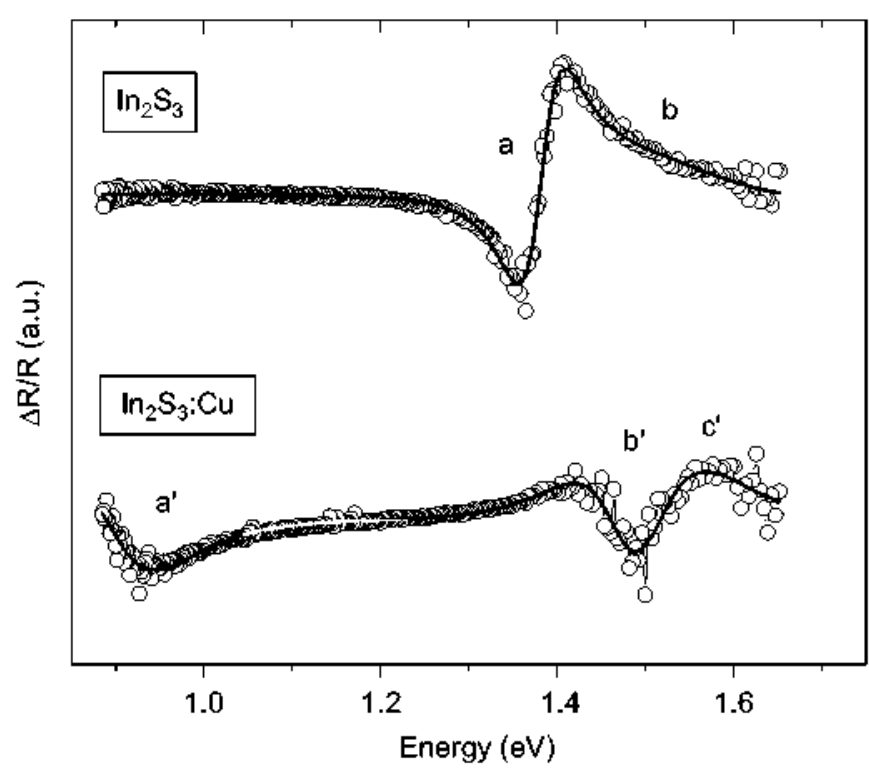

Fig. 5. PR spectra (open dots) of PVD-grown polycrystalline layers on Mo-coated glass (Na-free, Corning 7059 ) at $200^{\circ} \mathrm{C}$ : $\ln _{2} 5_{3}$ reference (top) and $\ln _{2} 5_{3}: \mathrm{Cu}$ (bottom). Solid lines represent TDFF fittings with parameters as summarized in Table 2.

Table 2

TDFF fitting parameters of spectra shown in Fig. 5 of $\ln _{2} S_{3}$ and $\ln _{2} 5: C u(|C u| \sim 2-3$ at: $)$ samples.

\begin{tabular}{lll}
\hline & $\ln _{2} 5_{3}$ & $\ln _{2} S_{3}: \mathrm{Cu}$ \\
\hline$E_{g, A}(\mathrm{eV})$ & 1.382 & 0.900 \\
$E_{g, B}(\mathrm{eV})$ & 1.520 & 1.482 \\
$E_{g, c}(\mathrm{eV})$ & - & 1.529 \\
$\Gamma_{a}(\mathrm{meV})$ & 41 & 96 \\
$\Gamma_{\mathrm{b}}(\mathrm{meV})$ & 208 & 72 \\
$\Gamma_{c}(\mathrm{meV})$ & - & 161 \\
$m_{a}$ & 1.80 & 1.96 \\
$m_{b}$ & 2.01 & 2.18 \\
$m_{c}$ & - & 2.16 \\
\hline
\end{tabular}

associated to native defects. It is well known that the properties of $\left[n_{2} S_{3}\right.$ are extremely sensitive to the growth conditions imposed and stoichiometric deviations, facilitated by a relative open crystalline structure of the spinel-type (see, for example Ref. [17] and refs. therein). In fact, one third of the tetrahedral sites of the cation sublattice remain vacant, and the different ordering of the vacancies determines two of the known structures of the compound. Furthermore, accurate control of the amount of sulphur incorporated into the structure is difficult to ensure, despite the fact that the chalcogen is typically provided in excess during the growth of the films.

Garlick et al. [18] reported luminescence spectra of crystalline $\left[n_{2} S_{3}\right.$ samples at $65 \mathrm{~K}$ in which two broad emissions were identified at 1.42 and $1.57 \mathrm{eV}$. The lack of structure of the broad lines was interpreted as the related recombination centers being well coupled to lattice vibrations. No conclusion was stated on the nature of the centers, but just a generic relation with the electron affinity of vacancies that may act as electron traps. We tentatively associate the PR-signatures found in our study with the reported PL emissions of Garlick et al. taking into account the energy shift associated to the low temperatures of the luminescence experiments.

With regard to the Cu-containing sample, Fig. 5 clearly shows that the incorporation of $\mathrm{Cu}$ modifies the optoelectronic properties of $\ln _{2} S_{3}\left[17 \mid\right.$. Features $a^{\prime}$ and $b^{\prime}$ are attributed, with certainty, to transitions related to deep centers. The a-signature of the reference sample acting as the embedding matrix in the Cu-containing sample is apparently suppressed. Transition $C^{t}$ at $1.53 \mathrm{eV}$ could, in principle, be attributed to the presence of $\mathrm{CuInS}_{2}$, assuming that $\mathrm{Cu}$ introduced during the growth of the films resulted in the formation of clusters of the ternary compound in the binary matrix. However, the similarities found in the fitting parameters of signatures $b$ and $c^{\prime}$ (all three showing nearly identical values) and particularly the large broadening factors, led us to consider a common origin for the critical points for both $\mathrm{Cu}$-free and Cu-containing samples as more likely.

Similar Cu-containing $\mathrm{In}_{2} \mathrm{~S}_{3}$ samples grown by PVD at low temperature have been recently studied by Barreau and Tessier [19|. The authors reported on the structural, optical and electrica] properties of the samples as a function of their $\mathrm{Cu}$-content. It is concluded that cu-atoms progressively occupy the vacant tetrahedral sites of the spinel-like $\ln _{2} S_{3}$ structure, distributing themselves homogeneously throughout the sample and reducing slightly the lattice parameters [20]. No evidence is reported in the work of Barreau of the formation of secondary phases related to ternary compounds, until the amount of Cu provided suffices for the segregation of $\mathrm{Cu}_{5} \mathrm{n}_{5} \mathrm{~S}_{8}$. Such an amount of $\mathrm{Cu}$ would correspond to a value $x=4$ according to the compositional formula [19]:

$\left[\mathrm{In}_{16}\right]_{\text {oh }}\left[\mathrm{ln}_{5.33-\{x / 3)} \mathrm{Cu}_{x} \square_{2.67-\{2 \times / 3\}}\right]_{\mathrm{Td}} S_{32}$

where $\square$ represents vacancies, and the subscripts $O$ h and $T d$ refer to sites of octahedral and tetrahedral coordination, respectively. For a total amount of $2-3$ at\% $\mathrm{Cu}$, the corresponding $x$-value is approximately 0.016 , which is well below the threshold value related to the formation of $\mathrm{CuIn}_{5} \mathrm{~S}_{8}$. The possibility of forming ternary clusters as a distinct phase other than $\left[n_{2} S_{3} \div C u\right.$ at Cu-concentration levels below $\sim 7$ at\% appears thus unlikely, although it cannot be discarded that local fluctuations in the $\mathrm{Cu}$ concentration could result in domains of $\mathrm{Cu}_{1} n_{5} \mathrm{~S}_{8}$ mixed with $\ln _{2} S_{3}: C u$, prior to the complete transformation of the layer into single-phase Culn $n_{5} S_{8}$. The fundamental transition of $\mathrm{CuIn} \mathrm{n}_{5} \mathrm{~S}_{8}$ is expected at about the same energy that the corresponding gap of CulnS $_{2}(1.52 \mathrm{eV})$. However, as discussed before, the results obtained for the reference $\ln _{2} \mathrm{~S}_{3}$ layer support the attribution of a common origin for the critical points $b$ and $c^{\prime}$ observed in the PR spectra. We thus cannot conclude with certainty about the presence of a distinct ternary phase.

\section{Conclusions}

In this work we have examined by means of optoelectronic characterization based on modulated photoreflectance two types of samples related to the family of Cu-containing chalcopyrites. These two types of samples are potential candidates for highefficiency photovoltaic devices and are under current study for the implementation of intermediate band solar cells. The idea of using this class of compounds in designs that profit from electronic confinement effects is appealing, though our results indicate that there are several technological barriers to their implementation. First, the embedding process of chalcopyrite nanostructures has to cope with the impact of cationic-interdiffusion processes occurring at standard processing temperatures, as revealed for the case of epitaxial layer stacks of selenide compounds. Even for samples grown at low temperatures, the abundance of native defects, and particularly the role played by native vacancies, may impose limits to the practical realization of devices based on sub-bandgap absorption, as observed for the case of polycrystalline sulphide structures. Despite these facts, we have demonstrated the suitability of modulated spectroscopic 
techniques for the characterization of nanostructured chalcopyrites and related materials.

\section{Acknowledgements}

This work has been partly supported by the Spanish National Program and Comunidad de Madrid (projects GÉNESIS-CSD2006/ 0004 and NUMANClA-S0505/ENE00310). D.F.M. acknowledges financial support from the Spanish Ministry of Science and Innovation within the program Ramón y Cajal. M.Y.L. acknowledges the support of the US National Science Foundation via International Research Fellowship award OlSE-0701460 administered by S. Parris.

\section{References}

[1] D. Fuertes Marrón, A. Marti, A. Luque, Thin-film intermediate band photovoltaics: advanced concepts for chalcopyrite solar cells, Phys. 5tatus Solidi A 206 (2009) 1021-1025.

[2] A. Luque, A. Marti, Increasing the efficiency of ideal solar cells by photon induced transitions at intermédiate levels, Plyss. Rev, lett. 78 (1997) 5014-5017.

3| A. Martí. D. Fuertes Marrón. A. luque, Evaluation of the efficiency potential of intermédiate band solar cells base don thin-film chalcopyrite materials, J. Appl. Phys. 103 (2008) 073706-6.

|4| I. Repins, M.A. Contreras, B. Egaas, C. DeHart, J. Scharf, C.L. Perkins, B. To, R. Noufi, $19.9 \%$-efficient ZnOtCdStCulnGaSe2 solar cell with $81.2 \%$ fill factor Prog. Photovolt. Res. Appl, 16 (2008) 235-239.

|5| V.S. Gurin. Nanoparticles of ternary semiconductors in colloids, low temperature formation and quantum size effects, Colloids Surf. A 142 (1998) 35-40. 5ee, for example;

M.A. Malik, P. O'Brien, N. Revaprasadu, A novel route for the preparation of CuSe and Culn5e2 nanoparticles, Adv, Mater. 11 (1999) 1441-1444;

C. Wang. 5. Xue, J. Hu, K. Tang. Raman, far-infrared and Mössbauer spectroscopy of CuFeS2 nanocrystallites, Jpn. J. Appl. Phys. 48 (2009) 023003-3;

S.L. Castro, S.G. Bailey, R.P. Raffaelle, K. Banger, A.F. Hepp, Nanocrystalline chalcopyrite materials Culn 52 and Culn5e2 via low-temperature pyrolysis of molecular single-source precursors. Chem. Mater. 15 (2003) 3142-3147.

[6] D. Fuertes Marrón, S. Lehmann, M.Ch. Lux-Steiner, Growth of isolated and embedded Cu-containing chalcopyrite cluster's and nanocrystals by diy processing. Phys. Rev. B 77 (2008) 085315-10;

D. Fuertes Marrón, S. Lehmann, J. Kosk, S. Sadewasser, M.Ch. Lux-Steiner, Growth and characterization of chalcopyrite nanocrystals: beyond conventional thin films, in: T. Gessert, S. Marsillac, T. Wada, K. Durose, and C. Heske (Eds.), MRS 5ymposia Proceedings 1012, MRS Warrendale, PA, 2007, Y02-07.
[7] M. Afshar, Wachstum und Charakterisierung von Multischicht Nanostrukturen aus CuGaSe2 und CulnSe2, Diploma Thesis, Freie Universität Berlin, 2008.

[8] N. Rega, S. Siebentritt, l. Beckers, J. Beckmann, ]. Albert, M.Ch. Lux-Steiner, MOVPE of epitaxial CulnSe2 on GaAs, J. Cryst. Growth 248 (2003) 169-174.

[9] J.E. Jaffe, A. Zunger, Defect-induced nonpolar-to-polar transition at the surface of chalcopyrite semiconductors, Phys. Rev. B 64 (2001) 241304R-4 D. Liao. A. Rockett. Epitaxial growth of Cu(In,Ga)Se2 on GaAs( 110$)$. J. Appl. Pliys, 91 (2002) 1978-1983.

[10] M. Cardona, Modulation Spectroscopy, Academic Press, New York, 1969 F.H. Pollak, H. Shen, Modulation spectroscopy of semiconductors: bulk/thin film. microstructures, surfacestinterfaces and devices, Mater. Sci. Eng. R10 (1993) 275-374;

0. Glembocki, B.V. Shanabrook, Semiconductors and Semimetals 36 (1992) 221-292.

[11] J.L. Shay, H.M. Kasper, Direct observation of Cu d-levels in 1-Il]-VI2 compounds, Phys, Rev, lett. 29 (1972) 1162-1164.

B. Tell, H.M. Kasper, Optical and electrical properties of AgGaS2 and AgGa5e2. Phys. Rev. B 4 (1971) 4455-4459;

J.L. Shay. J.H. Wernick, Ternary Chalcopyrite Semiconductors: Growth, Electronic Properties, and Applications, Pergamon Press, Oxford, 1975.

[12] S. Shirakata, S. Chichibu, Photoreflectance of Cu-based I-III-VI2 heteroepitaxial layer's grown by metalorganic chemical vapor deposition. J. Appl. Plyys. 79 (1996) 2043-2054

[13] D.E. Aspnes, Third derivative modulation spectroscopy with low field electroreflectance, Surf. Sci. 37 (1973) 418-442.

[14] N. Rega, Photolumineszenz von epitaktischen Cu(In,Ga) Se2 Schichten. Ph.D. Thesis, Freie Universität Berlin (2004): D.K. Suri, K.C. Nagpal, G.K. Cladha, X-ray study of CuGalnSe2 solid solutions, J. Appl. Crystallogr. 22 (1989) 578-583;

D. Abou-Ras, R. Caballero, C. Kaufmann, M. Nichterwitz, K. Sakurai, S. Schorr, T. Unold. H.W. Schock, Impact of the Ga concentration on the microstructure of CulnGaSe2. Pliys. 5tatus 5olidj (RRL) 2 (2008) 135-137.

[15] N. Rega, S. Siebentritt, J. Albert, M.Ch. Lux-Steiner, Photoluminescence of Cu(ln,Ga)Se2 epitaxial thin films grown by MOVPE, in: R. Noufi, W.N. Sluafarman. D. Cahen, l. 5tolt (Eds.). MRS Symposia Proceedings 763, MRS Warrendale, PA, 2003, B5-2.

[16] M.I. Alonso, M. Garriga, C.A. Durante Rincón, E. Hernández, M. León, Optica] functions of chalcopyrite CuGalnSe2 alloys, Appl. Phys. A 74 (2002) 659-664. S.-H. Han, D.H. Levi, H.A. Althani, F.S. Hasoon, R.N. Bhattacharya, A.M. Hermann, Optical functions of thin-film polycrystalline chalcopyrite CulnGaSe2, in: R. Noufi, W.N. Shafarman, D. Cahen, l. Stolt (Ed5.), MRS Symposia Proceedings 763, MRS Warrendale, PA, 2003, p. B1-8.

[17] N. Barreau, Indium sulphide and relatives in the world of photovoltaics, 501 Energy 83 (2009) 363-371.

[18] G.F.J. Garlick, M. Springford, H. Checinska, The infra-red emission of indium sesquisulphide, Proc. Phys. Soc. 82 (1963) 16-22.

[19] N. Barreau, M. Tessier, Characterization of indium sulfide thin films containing copper, in: A. Yamada, C. Heske, M. Contreras, M. Igalson, S.J.C. Irvine (Eds.), MRS Symposia Proceedings 1165. MR5 Warrendale, PA, 2009, M08-21

[20] F. Py, M. Womes, J.M. Durand, J. Oliver-Fourcade, J.C. Jumas, J.M. Esteva, R.C. Karnatak, Copper in In2S3: a study by X-ray diffraction, diffuse reflectance and X-ray absorption. J. Alloys Compd. 178 (1992) 297-304. 